\title{
Interim Estimates of 2016-17 Seasonal Influenza Vaccine Effectiveness - United States, February 2017
}

\begin{abstract}
Brendan Flannery, $\mathrm{PhD}^{1}$; Jessie R. Chung, $\mathrm{MPH}^{1}$; Swathi N. Thaker, $\mathrm{PhD}^{1}$; Arnold S. Monto, $\mathrm{MD}^{2}$; Emily T. Martin, PhD²; Edward A. Belongia, MD³; Huong Q. McLean, $\mathrm{PhD}^{3}$, Manjusha Gaglani, MBBS ${ }^{4}$; Kempapura Murthy, $\mathrm{MPH}^{4}$; Richard K. Zimmerman, MD ${ }^{5}$; Mary Patricia Nowalk, PhD ${ }^{5}$; Michael L. Jackson, $\mathrm{PhD}^{6}$; Lisa A. Jackson, $\mathrm{MD}^{6}$; Angie Foust, $\mathrm{MS}^{1}$; Wendy Sessions, $\mathrm{MPH}^{1}$; LaShondra Berman, $\mathrm{MS}^{1}$; Sarah Spencer, $\mathrm{PhD}^{1}$; Alicia M. Fry, $\mathrm{MD}^{1}$
\end{abstract}

In the United States, annual vaccination against seasonal influenza is recommended for all persons aged $\geq 6$ months (1). Each influenza season since 2004-05, CDC has estimated the effectiveness of seasonal influenza vaccine to prevent influenza-associated, medically attended, acute respiratory illness (ARI). This report uses data, as of February 4, 2017, from 3,144 children and adults enrolled in the U.S. Influenza Vaccine Effectiveness Network (U.S. Flu VE Network) during November 28, 2016-February 4, 2017, to estimate an interim adjusted effectiveness of seasonal influenza vaccine for preventing laboratory-confirmed influenza virus infection associated with medically attended ARI. During this period, overall vaccine effectiveness (VE) (adjusted for study site, age group, sex, race/ethnicity, self-rated general health, and days from illness onset to enrollment) against influenza $\mathrm{A}$ and influenza $\mathrm{B}$ virus infection associated with medically attended ARI was 48\% (95\% confidence interval $[\mathrm{CI}]=37 \%-57 \%)$. Most influenza infections were caused by $\mathrm{A}(\mathrm{H} 3 \mathrm{~N} 2)$ viruses. VE was estimated to be $43 \%$ $(\mathrm{CI}=29 \%-54 \%)$ against illness caused by influenza A (H3N2) virus and $73 \%(\mathrm{CI}=54 \%-84 \%)$ against influenza $\mathrm{B}$ virus. These interim $\mathrm{VE}$ estimates indicate that influenza vaccination reduced the risk for outpatient medical visits by almost half. Because influenza activity remains elevated (2), CDC and the Advisory Committee on Immunization Practices recommend that annual influenza vaccination efforts continue as long as influenza viruses are circulating (1). Vaccination with 2016-17 influenza vaccines will reduce the number of infections with most currently circulating influenza viruses. Persons aged $\geq 6$ months who have not yet been vaccinated this season should be vaccinated as soon as possible.

Methods used by the U.S. Flu VE Network have been published previously (3). At five study sites, patients aged $\geq 6$ months seeking outpatient medical care for an ARI with cough, within 7 days of illness onset, were enrolled.* Study enrollment began after $\geq 1$ laboratory-confirmed cases of influenza were identified

\footnotetext{
${ }^{*}$ The U.S. Flu VE Network sites and the date enrollment began are as follows: Group Health Cooperative (Seattle, Washington) (November 28, 2016); Marshfield Clinic Research Foundation (Marshfield, Wisconsin) (January 3, 2017); University of Michigan School of Public Health (the School of Public Health partnered with the University of Michigan Health System, Ann Arbor, and the Henry Ford Health System, Detroit, Michigan) (January 3, 2017); University of Pittsburgh Schools of the Health Sciences (the Schools of the Health Sciences partnered with the University of Pittsburgh Medical Center, Pittsburgh, Pennsylvania) (December 5, 2016); and Baylor Scott and White Health, Texas A\&M University Health Sciences Center College of Medicine (Temple, Texas) (December 13, 2016).
}

through local surveillance for $\geq 2$ consecutive weeks. Patients were eligible for enrollment if they 1) were aged $\geq 6$ months on September 1, 2016, and thus eligible for vaccination; 2) reported an ARI with cough and onset $\leq 7$ days earlier; and 3) had not been treated with influenza antiviral medication (e.g., oseltamivir) during this illness. After obtaining informed consent from patients or parents/guardians for their children, participants or their proxies were interviewed to collect demographic data, general and current health status, symptoms, and 2016-17 influenza vaccination status. Respiratory specimens were collected from each patient using nasal and oropharyngeal swabs, which were placed together in a single cryovial with viral transport medium. Only nasal swabs were collected for patients aged $<2$ years. Specimens were tested at U.S. Flu VE Network laboratories using CDC's real-time reverse transcription - polymerase chain reaction (rRT-PCR) protocol for detection and identification of influenza viruses. Participants (including children aged $<9$ years who require 2 vaccine doses during their first vaccination season) were considered vaccinated if they received $\geq 1$ dose of any seasonal influenza vaccine $\geq 14$ days before illness onset, according to medical records and registries (at Wisconsin site), medical records and self-report (at Texas and Washington sites), or selfreport only (Michigan and Pennsylvania sites). VE was estimated as $100 \% \mathrm{x}\left(1\right.$ - odds ratio). ${ }^{\dagger}$ Estimates were adjusted for study site, age group, sex, race/ethnicity, self-rated general health, and number of days from illness onset to enrollment using logistic regression. Interim VE estimates for the 2016-17 season were based on patients enrolled through February 4, 2017.

Among the 3,144 children and adults with ARI enrolled at the five study sites from November 28, 2016, through February 4, 2017, 744 (24\%) tested positive for influenza virus by rRT-PCR; 656 (88\%) of these viruses were influenza A, and $90(12 \%)$ were influenza B viruses (Table 1). Among 606 subtyped influenza A viruses, 595 (98\%) were A (H3N2) viruses. The proportion of patients with influenza differed by study site, sex, age group, race/ethnicity, and interval from illness onset to enrollment (Table 1). The proportion vaccinated ranged

\footnotetext{
$\dagger 100 \% \mathrm{x}$ (1 - odds ratio [ratio of odds of being vaccinated among outpatients with influenza-positive test results to the odds of being vaccinated among outpatients with influenza-negative test results]).
} 
TABLE 1. Selected characteristics for enrolled patients with medically attended acute respiratory illness, by influenza test result and seasonal influenza vaccination status - U.S. Influenza Vaccine Effectiveness Network, United States, November 28, 2016-February 4, 2017

\begin{tabular}{|c|c|c|c|c|c|c|}
\hline \multirow[b]{2}{*}{ Characteristic } & \multicolumn{2}{|c|}{ Influenza test result } & \multirow[b]{2}{*}{ p-value ${ }^{\dagger}$} & \multicolumn{2}{|c|}{ Vaccination status } & \multirow[b]{2}{*}{ p-value ${ }^{t}$} \\
\hline & No. positive (\%) & No. negative (\%) & & No. enrolled & No. vaccinated ${ }^{*}(\%)$ & \\
\hline Overall & $744(24)$ & $2,400(76)$ & & 3,144 & $1,650(52)$ & \\
\hline $\begin{array}{l}\text { State of study site } \\
\text { Michigan } \\
\text { Pennsylvania } \\
\text { Texas } \\
\text { Washington } \\
\text { Wisconsin }\end{array}$ & $\begin{array}{r}92(26) \\
176(30) \\
56(8) \\
374(38) \\
46(9)\end{array}$ & $\begin{array}{l}267(74) \\
416(70) \\
646(92) \\
613(62) \\
458(91)\end{array}$ & $<0.001$ & $\begin{array}{l}359 \\
592 \\
702 \\
987 \\
504\end{array}$ & $\begin{array}{l}206(57) \\
271(46) \\
341(49) \\
598(61) \\
234(46)\end{array}$ & $<0.001$ \\
\hline $\begin{array}{l}\text { Sex } \\
\text { Male } \\
\text { Female }\end{array}$ & $\begin{array}{l}350(26) \\
394(22)\end{array}$ & $\begin{array}{r}990(74) \\
1410(78)\end{array}$ & 0.005 & $\begin{array}{l}1,340 \\
1,804\end{array}$ & $\begin{array}{l}665(50) \\
985(55)\end{array}$ & 0.006 \\
\hline $\begin{array}{l}\text { Age group } \\
6 \text { mos- } 8 \text { yrs } \\
9-17 \text { yrs } \\
18-49 \text { yrs } \\
50-64 \text { yrs } \\
\geq 65 \text { yrs }\end{array}$ & $\begin{array}{r}97(14) \\
122(33) \\
208(21) \\
189(31) \\
128(28)\end{array}$ & $\begin{array}{l}614(86) \\
247(67) \\
783(79) \\
425(69) \\
331(72)\end{array}$ & $<0.001$ & $\begin{array}{l}711 \\
369 \\
991 \\
614 \\
459\end{array}$ & $\begin{array}{l}362(51) \\
128(35) \\
452(46) \\
337(55) \\
371(81)\end{array}$ & $<0.001$ \\
\hline $\begin{array}{l}\text { Race/Ethnicity } \\
\text { White } \\
\text { Black } \\
\text { Other race } \\
\text { Hispanic }\end{array}$ & $\begin{array}{r}532(23) \\
81(35) \\
72(24) \\
47(15)\end{array}$ & $\begin{array}{r}1,744(77) \\
153(65) \\
222(76) \\
274(85)\end{array}$ & $<0.001$ & $\begin{array}{r}2,276 \\
234 \\
294 \\
321\end{array}$ & $\begin{array}{r}1,231(54) \\
99(42) \\
163(55) \\
150(47)\end{array}$ & 0.001 \\
\hline $\begin{array}{l}\text { Self-rated health statu } \\
\text { Fair or poor } \\
\text { Good } \\
\text { Very good } \\
\text { Excellent }\end{array}$ & $\begin{array}{r}55(22) \\
179(23) \\
301(25) \\
209(23)\end{array}$ & $\begin{array}{l}200(78) \\
599(77) \\
902(75) \\
699(77)\end{array}$ & 0.52 & $\begin{array}{r}255 \\
778 \\
1,203 \\
908\end{array}$ & $\begin{array}{l}142(56) \\
436(56) \\
622(52) \\
450(50)\end{array}$ & 0.04 \\
\hline $\begin{array}{l}\text { Illness onset to enrollr } \\
<3 \\
3-4 \\
5-7\end{array}$ & $\begin{array}{l}284(29) \\
304(25) \\
156(17)\end{array}$ & $\begin{array}{l}693(71) \\
933(75) \\
774(83)\end{array}$ & $<0.001$ & $\begin{array}{r}977 \\
1,237 \\
930\end{array}$ & $\begin{array}{l}473(48) \\
654(53) \\
523(56)\end{array}$ & 0.003 \\
\hline $\begin{array}{l}\text { Influenza test result } \\
\text { Negative } \\
\text { Influenza B positiveף } \\
\text { B/Yamagata } \\
\text { B/Victoria } \\
\text { B lineage pending } \\
\text { Influenza A positiveף } \\
\text { A (H1N1)pdm09 } \\
\text { A (H3N2) } \\
\text { A subtype pending }\end{array}$ & $\begin{array}{r}- \\
90 \\
83 \\
4 \\
3 \\
656 \\
11 \\
595 \\
50\end{array}$ & $\begin{array}{r}2,400 \\
- \\
- \\
- \\
- \\
- \\
- \\
-\end{array}$ & - & $\begin{array}{r}2,400 \\
90 \\
83 \\
4 \\
3 \\
656 \\
11 \\
595 \\
50\end{array}$ & $\begin{array}{r}1,317(55) \\
23(26) \\
20(24) \\
1(25) \\
2(67) \\
310(47) \\
3(27) \\
282(47) \\
25(50)\end{array}$ & - \\
\hline
\end{tabular}

* Defined as having received $\geq 1$ dose of influenza vaccine $\geq 14$ days before illness onset. A total of 89 participants who received the vaccine $\leq 13$ days before illness onset were excluded from the study sample.

† The chi-square statistic was used to assess differences between the numbers of persons with influenza-negative and influenza-positive test results, in the distribution of enrolled patient and illness characteristics, and in differences between groups in the percentage vaccinated.

$\S$ Enrollees were categorized into one of four mutually exclusive racial/ethnic populations: white, black, other race, and Hispanic. Persons identified as Hispanic might have been of any race. Persons identified as white, black, or other race were non-Hispanic. Race/ethnicity data were missing for 19 enrollees.

9 Two patients had coinfection with influenza A and influenza B, making the sum 746, or two greater than the total number of influenza positives.

from $46 \%$ to $61 \%$ across sites and differed by sex, age group, and interval from illness onset to enrollment.

The proportion of ARI patients vaccinated with 2016-17 seasonal influenza vaccine was $45 \%$ among influenza patients compared with 55\% among influenza-negative participants (Table 2). After adjusting for study site, age group, sex, race/ ethnicity, self-rated general health, and number of days from illness onset to enrollment, VE against medically attended ARI because of influenza was $48 \%$ (CI $=37 \%-57 \%)$. VE for all ages was 43\% (CI $=29 \%-54 \%)$ against medically attended ARI because of $\mathrm{A}(\mathrm{H} 3 \mathrm{~N} 2)$ virus infection and $73 \%$ $(\mathrm{CI}=54 \%-84 \%)$ against influenza $\mathrm{B}$ virus infection. VE point estimates against $\mathrm{H} 3 \mathrm{~N} 2$-related illness varied by age group; statistically significant protection was found against H3N2related illness among children aged 6 months through 8 years $(\mathrm{VE}=53 \% ; \mathrm{CI}=16 \%-74 \%)$ and adults aged $50-64$ years $(\mathrm{VE}=50 \% ; \mathrm{CI}=23 \%-67 \%)$, whereas protection in other age groups did not reach statistical significance. 
TABLE 2. Number and percentage receiving 2016-17 seasonal influenza vaccine among 3,144 outpatients with acute respiratory illness and cough, by influenza test result status, age group, and vaccine effectiveness against all influenza $A$ and $B$ and against virus types $A$ (H3N2) and B - U.S. Influenza Vaccine Effectiveness Network, United States, November 28, 2016-February 4, 2017

\begin{tabular}{|c|c|c|c|c|c|c|}
\hline \multirow[b]{2}{*}{ Influenza type/Age group } & \multicolumn{2}{|c|}{ Influenza-positive } & \multicolumn{2}{|c|}{ Influenza-negative } & \multicolumn{2}{|c|}{ Vaccine effectiveness* } \\
\hline & Total & No. (\%) vaccinated & Total & No. (\%) vaccinated & Unadjusted \% (95\% Cl) & Adjusted \% $(95 \% \mathrm{Cl})$ \\
\hline \multicolumn{7}{|l|}{ Influenza $A$ and $B$} \\
\hline Overall & 744 & $333(45)$ & 2,400 & $1,317(55)$ & $33(21 \text { to } 44)^{\dagger}$ & $48(37 \text { to } 57)^{\dagger}$ \\
\hline \multicolumn{7}{|l|}{ Age group } \\
\hline $6 \mathrm{mos}-8 \mathrm{yrs}$ & 97 & $32(33)$ & 614 & $330(54)$ & $58(33 \text { to } 73)^{\dagger}$ & $53(22 \text { to } 72)^{\dagger}$ \\
\hline $9-17$ yrs & 122 & $36(30)$ & 247 & $92(37)$ & $29(-12$ to 56$)$ & 32 (-20 to 61$)$ \\
\hline $18-49$ yrs & 208 & $89(43)$ & 783 & $363(46)$ & 13 (-18 to 36$)$ & 19 (-17 to 43$)$ \\
\hline $50-64$ yrs & 189 & $76(40)$ & 425 & $261(61)$ & $58(40 \text { to } 70)^{\dagger}$ & $58(38 \text { to } 72)^{\dagger}$ \\
\hline$\geq 65 \mathrm{yrs}$ & 128 & $100(78)$ & 331 & $271(82)$ & 21 (-31 to 52$)$ & $46(4 \text { to } 70)^{\dagger}$ \\
\hline \multicolumn{7}{|l|}{ Influenza A (H3N2) } \\
\hline Overall & 595 & $282(47)$ & 2,400 & $1,317(55)$ & $26(11 \text { to } 38)^{\dagger}$ & $43(29 \text { to } 54)^{\dagger}$ \\
\hline \multicolumn{7}{|l|}{ Age group } \\
\hline 6 mos -8 yrs & 68 & $24(35)$ & 614 & $330(54)$ & $53(21 \text { to } 72)^{\dagger}$ & $53(16 \text { to } 74)^{\dagger}$ \\
\hline $9-17$ yrs & 94 & $28(30)$ & 247 & $92(37)$ & 29 (-19 to 57$)$ & 23 (-43 to 59$)$ \\
\hline $18-49$ yrs & 168 & $73(43)$ & 783 & $363(46)$ & 11 (-24 to 36$)$ & $13(-30$ to 41$)$ \\
\hline $50-64$ yrs & 154 & $70(45)$ & 425 & $261(61)$ & $48(24 \text { to } 64)^{\dagger}$ & $50(23 \text { to } 67)^{\dagger}$ \\
\hline$\geq 65$ yrs & 111 & 87 (78) & 331 & $271(82)$ & $20(-37$ to 53$)$ & $44(-3$ to 69$)$ \\
\hline \multicolumn{7}{|l|}{ Influenza B } \\
\hline Overall & 90 & $23(26)$ & 2,400 & $1,317(55)$ & $72(54 \text { to } 83)^{\dagger}$ & 73 (54 to 84$)^{\dagger}$ \\
\hline
\end{tabular}

Abbreviation: $\mathrm{Cl}=$ confidence interval.

* Vaccine effectiveness was estimated as $100 \%$ x ( 1 - odds ratio [ratio of odds of being vaccinated among outpatients with influenza-positive test results to the odds of being vaccinated among outpatients with influenza-negative test results]); odds ratios were estimated using logistic regression.

† Statistically significant at the $p<0.05$ level.

As of February 10, 2017, a total of 13 influenza A (H3N2) viruses from U.S. Flu VE Network participants had been characterized by CDC; 11 (85\%) belonged to genetic group 3C.2a or the related group 3C.2a1, and all of those characterized antigenically were similar to the reference virus representing the 2016-17 A (H3N2) vaccine component.

\section{Discussion}

Interim influenza vaccine effectiveness estimates for the 2016-17 season indicate that vaccination reduced the risk for influenza-associated medical visits by approximately half. Influenza activity is likely to continue for several more weeks in the United States, and vaccination efforts should continue as long as influenza viruses are circulating. Persons aged $\geq 6$ months who have not yet received the 2016-17 influenza vaccine should be vaccinated as soon as possible. ${ }^{\S}$ As of February 3, 2017, approximately 145 million doses of influenza vaccine had been distributed in the United States for the 2016-17 season.

Interim VE estimates indicate improved protection during the 2016-17 influenza season against the predominant influenza A (H3N2) virus belonging to genetic group 3C.2a, which emerged in early 2014 and was predominant during the 2014-15 influenza season in the United States. During 2014-15, these

\footnotetext{
$\$$ A local influenza vaccine provider can be found by accessing the Flu Vaccine

Finder website at https://vaccinefinder.org/?address.
}

influenza A (H3N2) 3C.2a viruses were antigenically different from the recommended $\mathrm{A}(\mathrm{H} 3 \mathrm{~N} 2)$ vaccine component, and this resulted in low $(1 \%)$ vaccine effectiveness against illness caused by influenza A (H3N2) 3C.2a viruses (4). Low effectiveness of the 2014-15 vaccines likely contributed to high rates of influenza-associated hospitalizations that season, especially among adults aged $\geq 65$ years. In contrast, rates of influenza-associated hospitalizations observed to date have been substantially lower during the 2016-17 season (2). Virologic surveillance indicates that the majority of influenza A $(\mathrm{H} 3 \mathrm{~N} 2)$ viruses collected by U.S. laboratories during the 2016-17 season remain antigenically similar to the A/Hong Kong/4801/2014-like cell propagated reference virus belonging to genetic group $3 \mathrm{C} .2 \mathrm{a}$, which is the recommended influenza A (H3N2) component of the 2016-17 Northern Hemisphere vaccine.

Since the 2009 influenza A (H1N1) pandemic, VE estimates for $A(H 3 N 2)$ viruses have been lower than VE estimates against A (H1N1) and influenza B viruses. Interim VE estimates against illness caused by influenza A (H3N2) viruses during the 2016-17 influenza season are similar to U.S. VE estimates against A (H3N2)-related illness during the 2011-12 and 2012-13 seasons $(\mathrm{VE}=39 \%)(5,6)$. Also, a meta-analysis of VE studies using the test-negative design conducted from the 2007-08 through the 2014-15 influenza seasons reported a pooled VE estimate against A (H3N2)-related illness of 33\% $(\mathrm{CI}=26 \%-39 \%)$, compared with $61 \%(\mathrm{CI}=57 \%-65 \%)$ 
against influenza $\mathrm{A}(\mathrm{H} 1 \mathrm{~N} 1) \mathrm{pdm} 09$ and 54\% (CI = 46\%-61\%) against influenza $\mathrm{B}$ virus-related illness ( 7 ). These results reflect properties unique to $A(\mathrm{H} 3 \mathrm{~N} 2)$ viruses that pose special challenges. Influenza A (H3N2) viruses undergo more frequent and extensive genetic changes than do influenza A (H1N1) and influenza $\mathrm{B}$ viruses, and require more frequent updates to the $\mathrm{A}(\mathrm{H} 3 \mathrm{~N} 2)$ vaccine virus components to maintain activity against evolving circulating strains. In addition, A (H3N2) viruses continue to undergo changes in their receptor-binding specificity, which might result in genetic changes during growth in eggs. Most influenza vaccines are manufactured using eggbased production processes. These genetic changes (referred to as egg-adapted changes) alter the antigenic properties of candidate vaccine viruses (CVVs) as they are grown in eggs and potentially during the vaccine production process $(8)$. The egg-adapted changes might contribute to the lower vaccine effectiveness seen with $A(\mathrm{H} 3 \mathrm{~N} 2)$ viruses compared with $A(\mathrm{H} 1 \mathrm{~N} 1)$ and $B$ viruses. Efforts are ongoing to improve influenza vaccine effectiveness against $\mathrm{A}(\mathrm{H} 3 \mathrm{~N} 2)$ viruses in $\mathrm{CVV}$ development and in manufacturing.

As of February 10, 2017, influenza activity remained elevated nationally and was widespread across most of the United States. During recent A (H3N2) virus predominant-seasons, persons aged $\geq 65$ years and young children experienced higher rates of severe illness and influenza-associated hospitalization compared with other age groups. With vaccine effectiveness of $48 \%$, some vaccinated persons will become infected with influenza. Clinicians should maintain a high index of suspicion for influenza infection among persons with acute respiratory illness while influenza activity is ongoing, especially among older adults. Early antiviral treatment can reduce severity and complications of influenza-associated illness (9). Early antiviral treatment is recommended for persons with suspected influenza with severe or progressive illness (e.g., hospitalized persons) and persons at high risk for complications from influenza, such as children aged $<2$ years, adults aged $\geq 65$ years and persons with underlying health conditions, 9 even if illness is less severe.

\footnotetext{
A complete summary of guidance for antiviral use is available at https://www. cdc.gov/flu/professionals/antivirals/summary-clinicians.htm. Groups at high risk for influenza complications include the following: children aged <2 years; adults aged $\geq 65$ years; persons with chronic pulmonary conditions (including asthma); cardiovascular disease (except hypertension alone); persons with renal, hepatic, or hematologic (including sickle cell) disease; persons with metabolic disorders (including diabetes mellitus); persons with neurologic and neurodevelopmental conditions (including disorders of the brain, spinal cord, peripheral nerves and muscles, such as cerebral palsy, epilepsy [seizure disorders], stroke, intellectual disability [mental retardation], moderate to severe developmental delay, muscular dystrophy, or spinal cord injury); persons with immunosuppression, including that caused by medications or by human immunodeficiency virus infection; women who are pregnant or postpartum (within 2 weeks after delivery); persons aged $<19$ years who are receiving long-term aspirin therapy; American Indian/ Alaska Natives; persons with morbid obesity (i.e., body-mass index $\geq 40$ ); and residents of nursing homes and other chronic-care facilities.
}

Antiviral medications should be used as recommended for treatment in patients with suspected influenza, regardless of vaccination status. The decision to initiate antiviral treatment should not be delayed while waiting for laboratory confirmation of influenza and should not be dependent on insensitive assays, such as rapid influenza diagnostic tests.

The findings in this report are subject to at least four limitations. First, vaccination status included self-report at four of five sites. End-of-season VE estimates based on updated documentation of vaccination status might differ from interim estimates. Second, information from medical records and immunization registries is needed to evaluate $\mathrm{VE}$ by vaccine type and for fully vaccinated compared with partially vaccinated children (children aged $<9$ years require 2 vaccine doses during their first vaccination season), as well as to evaluate the effects of prior season vaccination and timing of vaccination; end-of-season analysis of VE by vaccine type and effects of partial or prior season vaccination is planned. Third, an observational study design has greater potential for confounding and bias relative to randomized clinical trials. However, the test-negative design is widely used in VE studies and has been used by the U.S. Flu VE Network to estimate VE for the past several influenza seasons. Finally, small sample sizes in some age groups resulted in wide confidence intervals, and end-of-season VE estimates could change as additional patient data become available or if there is a change in circulating viruses late in the season. It is also important to note that the VE estimates in this report are limited to the prevention of outpatient medical visits, rather than more severe illness outcomes, such as hospitalization or death; data from studies measuring VE against more severe outcomes will be available at a later date.

Annual vaccination against circulating influenza viruses remains the best strategy for preventing illness from influenza. As of early November 2016, only $37 \%$ of children aged 6 months-17 years, $37 \%$ of adults aged 18-64 years, and $57 \%$ of adults aged $\geq 65$ years had received influenza vaccine this season (10). Among pregnant women, early estimates for $2016-17$ indicated that only $47 \%$ had been vaccinated by early November 2016 (10). In addition to ongoing vaccination efforts, antiviral medications continue to be an important adjunct to the treatment and control of influenza and should be used as recommended, regardless of patient vaccination status.

\section{Acknowledgments}

Erika Kiniry, Stacie Wellwood, C. Hallie Phillips, Suzie Park, Lawrence Madziwa, Matt Nguyen, Group Health Research Institute, Seattle, Washington; Jennifer K. Meece, Jennifer P. King, Elizabeth Armagost, Deanna Cole, Terry Foss, Dyan Friemoth, Katherine Graebel-Khandakani, Linda Heeren, Tami Johnson, Tara Johnson, Nicole Kaiser, Diane Kohnhorst, Sarah Kopitzke, Ariel Marcoe, 
Karen McGreevey, Madalyn Minervini, Vicki Moon, Suellyn Murray, Rebecca Pilsner, DeeAnn Polacek, Emily Redmond, Miriah Rotar, Carla Rottscheit, Jacklyn Salzwedel, Samantha Smith, Sandra Strey, Jane Wesely, Lynn Ivacic, Sherri Guzinski, Jennifer Anderson, Klevi Hoxha, Tammy Koepel, Nan Pan, Annie Steinmetz, Gregg Greenwald, Marshfield Clinic Research Foundation, Marshfield, Wisconsin; Joshua G. Petrie, Lois E. Lamerato, Ryan E. Malosh, E.J. McSpadden, Hannah Segaloff, Caroline K. Cheng, Rachel Truscon, Emileigh Johnson, Anne Kaniclides, Heather R. Lipkovich, Nishat Islam, Michelle Groesbeck, Andrea Lee, Joey Lundgren, Erika Chick, Lindsey Benisatto, Tosca Le, Dexter Hobdy, Kristyn Brundidge, Christina Rincon, Stephanie Haralson, Jennifer Hessen, Ahn Trinh, University of Michigan, Ann Arbor, and Henry Ford Health System, Detroit, Michigan; John V. Williams, Monika Johnson, Todd M. Bear, Heather Eng, Samantha Ford, Krissy K. Moehling, Jonathan M. Raviotta, Sean Saul, Terrie Sax, Michael Susick, G.K. Balasubramani, PhD, Rina Chabra, MD, Edward Garofolo, MD, Philip Iozzi, MD, Barbara Kevish, MD, Donald B. Middleton, MD, Christopher Olbrich, MD, Evelyn C. Reis, MD, Leonard Urbanski, MD, University of Pittsburgh Schools of the Health Sciences and University of Pittsburgh Medical Center, Pittsburgh, Pennsylvania; Anne Robertson, Ashley Kossie, Michael Smith, Vanessa Hoelscher, Lydia Clipper, Kevin Dunlap, Crystal Hodges, Teresa Ponder, Ineshia Jackson, Deborah Furze, Mary Kylberg, Martha Zayed, Melissa Zdroik, Kimberley Walker, Marcus Volz, Arundhati Rao, Robert Fader, Yolanda Munoz-Maldonado, Lea Mallett, Hania Wehbe-Janek, Madhava Beeram, Michael Reis, Jennifer Thomas, Jaime Walkowiak, Jeremy Ray, Renee Day, Deborah Price, Jennifer Fox, Baylor Scott and White Health, Texas A\&M University Health Sciences Center College of Medicine, Temple, Texas; Erin Burns, MA, Elisabeth Blanchard, Priya Budhathoki, Thomas Rowe, Lizheng Guo, Influenza Division, National Center for Immunization and Respiratory Diseases, CDC.

\footnotetext{
${ }^{1}$ Influenza Division, National Center for Immunization and Respiratory Diseases, CDC; ${ }^{2}$ University of Michigan, Ann Arbor, Michigan; ${ }^{3}$ Marshfield Clinic Research Foundation, Marshfield, Wisconsin; ${ }^{4}$ Baylor Scott and White Health, Texas A\&M University Health Science Center College of Medicine, Temple, Texas; ${ }^{5}$ University of Pittsburgh Schools of the Health Sciences and University of Pittsburgh Medical Center, Pittsburgh, Pennsylvania; ${ }^{6}$ Group Health Research Institute, Seattle, Washington.
}

Corresponding author: Brendan Flannery, bif4@cdc.gov, 404-718-4276.

\section{References}

1. Grohskopf LA, Sokolow LZ, Broder KR, et al. Prevention and control of seasonal influenza with vaccines: recommendations of the Advisory Committee on Immunization Practices-United States, 2016-17 influenza season. MMWR Recomm Rep 2016;65(No. RR-5). http:// dx.doi.org/10.15585/mmwr.rr6505a1

2. CDC. Fluview. 2016-2017 influenza season week 6 ending February 11, 2017. Atlanta, GA: US Department of Health and Human Services, CDC; 2017. https://www.cdc.gov/flu/weekly

3. Zimmerman RK, Nowalk MP, Chung J, et al. 2014-2015 influenza vaccine effectiveness in the United States by vaccine type. Clin Infect Dis 2016;63:1564-73. http://dx.doi.org/10.1093/cid/ciw635

4. Flannery B, Zimmerman RK, Gubareva LV, et al. Enhanced genetic characterization of influenza $\mathrm{A}(\mathrm{H} 3 \mathrm{~N} 2)$ viruses and vaccine effectiveness by genetic group, 2014-2015. J Infect Dis 2016;214:1010-9. http:// dx.doi.org/10.1093/infdis/jiw181

5. Ohmit SE, Thompson MG, Petrie JG, et al. Influenza vaccine effectiveness in the 2011-2012 season: protection against each circulating virus and the effect of prior vaccination on estimates. Clin Infect Dis 2014;58:319-27. http://dx.doi.org/10.1093/cid/cit736

6. McLean HQ, Thompson MG, Sundaram ME, et al. Influenza vaccine effectiveness in the United States during 2012-2013: variable protection by age and virus type. J Infect Dis 2015;211:1529-40. http://dx.doi. org/10.1093/infdis/jiu647

7. Belongia EA, Simpson MD, King JP, et al. Variable influenza vaccine effectiveness by subtype: a systematic review and meta-analysis of testnegative design studies. Lancet Infect Dis 2016;16:942-51. http:// dx.doi.org/10.1016/S1473-3099(16)00129-8

8. Barr IG, Russell C, Besselaar TG, et al. WHO recommendations for the viruses used in the 2013-2014 Northern Hemisphere influenza vaccine: epidemiology, antigenic and genetic characteristics of influenza $\mathrm{A}(\mathrm{H} 1 \mathrm{~N} 1)$ pdm09, $\mathrm{A}(\mathrm{H} 3 \mathrm{~N} 2)$ and $\mathrm{B}$ influenza viruses collected from October 2012 to January 2013. Vaccine 2014;32:4713-25. http://dx.doi.org/10.1016/j. vaccine.2014.02.014

9. Fiore AE, Fry A, Shay D, Gubareva L, Bresee JS, Uyeki TM. Antiviral agents for the treatment and chemoprophylaxis of influenzarecommendations of the Advisory Committee on Immunization Practices (ACIP). MMWR Recomm Rep 2011;60(No. RR-1).

10. CDC. FluVaxView. Influenza vaccination coverage. Atlanta, GA: US Department of Health and Human Services, CDC; 2016. https://www. cdc.gov/flu/fluvaxview/index.htm 